\title{
The Equivalence of Datko and Lyapunov Properties for $(h, k)$-Trichotomic Linear Discrete-Time Systems
}

\author{
Claudia-Luminiţa Mihiţ, ${ }^{1}$ Mihail Megan, ${ }^{1,2}$ and Traian Ceauşu ${ }^{1}$ \\ ${ }^{1}$ Department of Mathematics, Faculty of Mathematics and Computer Science, West University of Timişoara, \\ Vasile Pârvan Boulevard No. 4, 300223 Timişoara, Romania \\ ${ }^{2}$ Academy of Romanian Scientists, Independenţei 54, 050094 Bucharest, Romania
}

Correspondence should be addressed to Mihail Megan; megan@math.uvt.ro

Received 22 July 2015; Accepted 26 January 2016

Academic Editor: Allan C. Peterson

Copyright (c) 2016 Claudia-Luminiţa Mihiţ et al. This is an open access article distributed under the Creative Commons Attribution License, which permits unrestricted use, distribution, and reproduction in any medium, provided the original work is properly cited.

The aim of this paper is to characterize a general property of $(h, k)$-trichotomy through some Lyapunov functions for linear discrete-time systems in infinite dimensional spaces. Also, we apply the results to illustrate necessary and sufficient conditions for nonuniform exponential trichotomy and nonuniform polynomial trichotomy.

\section{Introduction}

In the last few years an important development has been made in the field of the asymptotic behaviors of dynamical systems. Among the most important asymptotic behaviors studied, we mention the properties of stability, dichotomy, and trichotomy (see [1-14] and the references therein).

A remarkable characterization for the stability property of continuous dynamical systems was proved by Datko in 1972 (see [8]) and later, Przyłuski and Rolewicz obtain in [15] a similar result for discrete-time systems. This was a starting point for the development of the area and the results were extended to the dichotomy case in $[16,17]$.

An important generalization of the dichotomy concept (approached in various manners in $[2,3,6,18]$ ) is the notion of trichotomy, the most complex asymptotic property of dynamical systems. The trichotomy supposes the splitting of the state space, at any moment, into three subspaces: the stable subspace, the unstable subspace, and the central subspace.

The concept of (exponential) trichotomy was introduced by Elaydi and Hajek (see $[9,10]$ ) for nonlinear differential equations and later, the case of difference equations is treated by Elaydi and Janglajew in [11]. Also, important contributions on the line of trichotomy in discrete-time are due to Cuevas and Vidal [7], López-Fenner and Pinto [13], Megan and Stoica $[19,20]$, Papaschinopoulos [21], and Popa et al. [22].

In [23], A. L. Sasu and B. Sasu propose an interesting technique for exponential trichotomy of difference equations, the admissibility technique, and in [24] the authors obtain for the first time nonlinear conditions for the exponential trichotomy in infinite dimensional spaces.

The Lyapunov functions represent an important tool in the study of the asymptotic properties of dynamical systems (see, e.g., $[4,5,25,26]$ ).

The objective of this paper is to approach the general concept of $(h, k)$-trichotomy, where $h$ and $k$ are growth rates, for linear discrete-time systems in Banach spaces and as particular cases we deduce the results for (nonuniform) exponential trichotomy and (nonuniform) polynomial trichotomy.

Also, we obtain necessary and sufficient conditions for a general concept of $(h, k)$-trichotomy (called $(h, k)$ trichotomy of Datko type) and the main result is the characterization of this concept of trichotomy in terms of Lyapunov functions. 
The results are applied to illustrate criteria through the Lyapunov functions for nonuniform exponential trichotomy and nonuniform polynomial trichotomy.

\section{Growth Rates}

Definition 1. An increasing sequence $h: \mathbb{N} \rightarrow[1,+\infty)$, $h(n)=h_{n}$ is called a growth rate if $\lim _{n \rightarrow \infty} h_{n}=+\infty$.

Definition 2. One says that the growth rate $\left(h_{n}\right)$ satisfies hypothesis $(\mathscr{H})$ if there exist a growth rate $\left(f_{n}\right)$ and $M \in$ $(1,+\infty)$ such that

$\left(H_{1}\right)$

$$
\sum_{n=0}^{+\infty} \frac{f_{n}}{h_{n}} \leq M
$$

$\left(H_{2}\right)$

$$
\sum_{j=n}^{m-1} \frac{h_{j}}{f_{j}} \leq M \frac{h_{m}^{2}}{f_{m}^{2}}
$$

for all $(m, n) \in \mathbb{N} \times \mathbb{N}, m>n$.

Now, we present some examples of growth rates which satisfy hypothesis $(\mathscr{H})$.

Example 3. Let $h_{n}=e^{n \alpha}, \alpha>0$, and $f_{n}=e^{n \beta} ; \beta \in(0, \alpha)$ is a growth rate with

$\left(H_{1}\right)$

$$
\sum_{n=0}^{+\infty} \frac{f_{n}}{h_{n}}=\sum_{n=0}^{+\infty} e^{n(\beta-\alpha)}=\frac{e^{\alpha}}{e^{\alpha}-e^{\beta}}=M
$$

$\left(H_{2}\right)$

$$
\begin{aligned}
\sum_{j=n}^{m-1} \frac{h_{j}}{f_{j}} & =\sum_{j=n}^{m-1} e^{j(\alpha-\beta)}=\frac{e^{(\alpha-\beta) m}-e^{n(\alpha-\beta)}}{e^{\alpha-\beta}-1} \\
& \leq \frac{e^{\alpha}}{e^{\alpha}-e^{\beta}} e^{2 m(\alpha-\beta)}=M \frac{h_{m}^{2}}{f_{m}^{2}},
\end{aligned}
$$

for all $(m, n) \in \mathbb{N}^{2}, m>n$.

Example 4. If $h_{n}=(n+1)^{\alpha}$ with $\alpha>1$, then $f_{n}=(n+1)^{\beta-1}$ with $\beta \in(1, \alpha)$ is a growth rate which satisfies the following:

$\left(H_{1}\right)$

$$
\begin{aligned}
& \sum_{n=0}^{+\infty} \frac{f_{n}}{h_{n}}=\sum_{n=0}^{+\infty}(n+1)^{\beta-1-\alpha}=\sum_{n=0}^{+\infty} \frac{1}{(n+1)^{\alpha-\beta+1}} \\
&=M \in(1,+\infty) ; \\
&\left(H_{2}\right) \quad \\
& \sum_{j=n}^{m-1} \frac{h_{j}}{f_{j}}=\sum_{j=n}^{m-1}(j+1)^{\alpha-\beta+1} \leq(m-n)(m+1)^{\alpha-\beta+1} \\
& \leq(m+1)^{2(\alpha-\beta+1)}=\frac{h_{m}^{2}}{f_{m}^{2}} \leq M \frac{h_{m}^{2}}{f_{m}^{2}},
\end{aligned}
$$

for all $(m, n) \in \mathbb{N}^{2}, m>n$.
Example 5. Let $h_{n}=(n+1)^{\alpha} e^{n \gamma}$ with $\alpha>1$ and $\gamma>0$. Then $f_{n}=(n+1)^{\beta-1} e^{\delta n}$ with $\beta \in(1, \alpha), \delta \in(0, \gamma)$ is a growth rate with the following properties:

$\left(H_{1}\right)$

$$
\sum_{n=0}^{+\infty} \frac{f_{n}}{h_{n}}=\sum_{n=0}^{+\infty} \frac{e^{n(\delta-\gamma)}}{(n+1)^{\alpha-\beta+1}} \leq \sum_{n=0}^{+\infty} e^{n(\delta-\gamma)}=\frac{e^{\gamma}}{e^{\gamma}-e^{\delta}}=M ;
$$

$\left(\mathrm{H}_{2}\right)$

$$
\begin{aligned}
\sum_{j=n}^{m-1} \frac{h_{j}}{f_{j}} & =\sum_{j=n}^{m-1}(j+1)^{\alpha-\beta+1} e^{j(\gamma-\delta)} \\
& \leq(m-n)(m+1)^{\alpha-\beta+1} e^{(\gamma-\delta) m} \\
& \leq(m+1)^{2(\alpha-\beta+1)} e^{2(\gamma-\delta) m}=\frac{h_{m}^{2}}{f_{m}^{2}} \leq M \frac{h_{m}^{2}}{f_{m}^{2}},
\end{aligned}
$$

for all $(m, n) \in \mathbb{N}^{2}, m>n$.

\section{3. $(h, k)$-Trichotomy}

Let $X$ be a real or complex Banach space and $\mathscr{B}(X)$ the Banach algebra of all bounded linear operators on $X$. I represents the identity operator on $X$ and the norms on $X$ and on $\mathscr{B}(X)$ will be denoted by $\|\cdot\|$. Also,

$$
\Delta=\left\{(m, n) \in \mathbb{N}^{2}: m \geq n\right\},
$$

where $\mathbb{N}$ is the set of nonnegative integers.

We consider the linear discrete-time system

$$
x_{n+1}=A_{n} x_{n}, \quad n \in \mathbb{N},
$$

with $A: \mathbb{N} \rightarrow \mathscr{B}(X), A(n)=A_{n}$.

Every solution of $(\mathscr{A})$ is given by

$$
x_{m}=A_{m}^{n} x_{n},
$$

for all $(m, n) \in \Delta$, where

$$
A_{m}^{n}:= \begin{cases}A_{m-1}, \ldots, A_{n}, & \text { if } m>n \\ I, & \text { if } m=n .\end{cases}
$$

Remark 6. We observe that

$$
A_{m}^{n} A_{n}^{p}=A_{m}^{p},
$$

for all $(m, n),(n, p) \in \Delta$.

Definition 7. A sequence $P: \mathbb{N} \rightarrow \mathscr{B}(X), P(n)=P_{n}$ is called a projections sequence on $X$ if

$$
P_{n}^{2}=P_{n},
$$

for all $n \in \mathbb{N}$. 
Definition 8. One says that $\mathscr{P}=\left\{P_{n}^{1}, P_{n}^{2}, P_{n}^{3}\right\}$ is a family of

(i) supplementary projections sequences if $\left(s_{1}\right)$

$\left(s_{2}\right)$

$$
P_{n}^{1}+P_{n}^{2}+P_{n}^{3}=I, \quad \forall n \in \mathbb{N}
$$

$$
P_{n}^{i} P_{n}^{j}=0, \quad \forall i \neq j, \quad i, j \in\{1,2,3\}
$$

(ii) invariant projections sequences for $(\mathscr{A})$ if

$$
P_{n+1}^{i} A_{n}=A_{n} P_{n}^{i}, \quad \forall n \in \mathbb{N}, i \in\{1,2,3\} .
$$

In what follows, we consider $h, k: \mathbb{N} \rightarrow[1,+\infty)$ two growth rates and a pair $(\mathscr{A}, \mathscr{P})$, where $\mathscr{P}=\left\{P_{n}^{1}, P_{n}^{2}, P_{n}^{3}\right\}$ is a family of supplementary and invariant projections sequences for $(\mathscr{A})$.

Definition 9. The pair $(\mathscr{A}, \mathscr{P})$ is called $(h, k)$-trichotomic if there exists a nondecreasing sequence $\left(s_{n}\right), s_{n} \geq 1$, such that

$\left(h t_{1}\right)$

$$
h_{m}\left\|A_{m}^{n} P_{n}^{1} x\right\| \leq h_{n} s_{n}\left\|P_{n}^{1} x\right\|
$$

$\left(h t_{2}\right)$

$$
h_{m}\left\|P_{n}^{2} x\right\| \leq h_{n} s_{m}\left\|A_{m}^{n} P_{n}^{2} x\right\|
$$

$\left(k t_{3}\right)$

$$
k_{n}\left\|A_{m}^{n} P_{n}^{3} x\right\| \leq k_{m} s_{n}\left\|P_{n}^{3} x\right\|
$$

$\left(k t_{4}\right)$

$$
k_{n}\left\|P_{n}^{3} x\right\| \leq k_{m} s_{m}\left\|A_{m}^{n} P_{n}^{3} x\right\|
$$

for all $(m, n, x) \in \Delta \times X$.

In the particular case when $\left(s_{n}\right)$ is a constant sequence, $(\mathscr{A}, \mathscr{P})$ is called uniformly $(h, k)$-trichotomic.

As particular cases of $(h, k)$-trichotomy we remark the following:

(i) if $h_{n}=e^{n \alpha}, k_{n}=e^{n \beta}$ with $\alpha, \beta>0$ we obtain the concept of (nonuniform) exponential trichotomy and if $\left(s_{n}\right)$ is constant it results in the property of uniform exponential trichotomy;

(ii) if $h_{n}=(n+1)^{\alpha}, k_{n}=(n+1)^{\beta}$ with $\alpha, \beta>1$ we recover the concept of (nonuniform) polynomial trichotomy and if $\left(s_{n}\right)$ is constant it results in the property of uniform polynomial trichotomy;

(iii) if $P_{n}^{3}=0$ for all $n \in \mathbb{N}$ it results in the notion of $h$ dichotomy, nonuniform exponential dichotomy (for $h_{n}=e^{n \alpha}, \alpha>0$ ), uniform exponential dichotomy (for $h_{n}=e^{n \alpha}, \alpha>0$, and $\left(s_{n}\right)$ constant), nonuniform polynomial dichotomy (for $\left.h_{n}=(n+1)^{\alpha}, \alpha>1\right)$, and uniform polynomial dichotomy (for $h_{n}=(n+1)^{\alpha}$, $\alpha>1$, and $\left(s_{n}\right)$ constant).

We give a general example of a pair $(\mathscr{A}, \mathscr{P})$ which is $(h, k)$ trichotomic.
Example 10. Let $\left(h_{n}\right)$ and $\left(k_{n}\right)$ be two growth rates and $\left(s_{n}\right)$ a nondecreasing sequence of positive real numbers, $s_{n} \geq 1$.

Let $\mathscr{P}=\left\{P_{n}^{1}, P_{n}^{2}, P_{n}^{3}\right\}$ be a family of supplementary projections sequences with

$$
P_{n+1}^{i} P_{n}^{i}=P_{n}^{i}, \quad \forall n \in \mathbb{N}, i=1,2,3 .
$$

Linear discrete-time system $(\mathscr{A})$, defined by

$$
A_{n}=\frac{h_{n} s_{n}}{h_{n+1} s_{n+1}} P_{n}^{1}+\frac{h_{n+1} s_{n+1}}{h_{n} s_{n}} P_{n}^{2}+\frac{k_{n+1} s_{n}}{k_{n} s_{n+1}} P_{n}^{3},
$$

verifies the relation

$$
P_{n+1}^{i} A_{n}=A_{n} P_{n}^{i}, \quad i=1,2,3 .
$$

Then

$$
A_{m}^{n}=\frac{h_{n} s_{n}}{h_{m} s_{m}} P_{n}^{1}+\frac{h_{m} s_{m}}{h_{n} s_{n}} P_{n}^{2}+\frac{k_{m} s_{n}}{k_{n} s_{m}} P_{n}^{3},
$$

for all $(m, n) \in \Delta$.

For all $(m, n, x) \in \Delta \times X$ the following properties hold:

(i)

$$
h_{m}\left\|A_{m}^{n} P_{n}^{1} x\right\|=h_{n} \frac{s_{n}}{s_{m}}\left\|P_{n}^{1} x\right\| \leq h_{n} s_{n}\left\|P_{n}^{1} x\right\| ;
$$

(ii)

$$
h_{m}\left\|P_{n}^{2} x\right\| \leq h_{m} \frac{s_{m}^{2}}{s_{n}}\left\|P_{n}^{2} x\right\|=h_{n} s_{m}\left\|A_{m}^{n} P_{n}^{2} x\right\| ;
$$

(iii)

$$
k_{n}\left\|A_{m}^{n} P_{n}^{3} x\right\|=k_{m} \frac{s_{n}}{s_{m}}\left\|P_{n}^{3} x\right\| \leq k_{m} s_{n}\left\|P_{n}^{3} x\right\| ;
$$

(iv)

$$
k_{n}\left\|P_{n}^{3} x\right\| \leq \frac{k_{m}^{2}}{k_{n}} s_{n}\left\|P_{n}^{3} x\right\|=k_{m} s_{m}\left\|A_{m}^{n} P_{n}^{3} x\right\|
$$

and we deduce that the pair $(\mathscr{A}, \mathscr{P})$ is $(h, k)$-trichotomic.

Remark 11. It is obvious that if the pair $(\mathscr{A}, \mathscr{P})$ is uniformly $(h, k)$-trichotomic then it is also $(h, k)$-trichotomic. In the following example we show that the converse implication is not valid.

Example 12. We consider $\mathscr{P}=\left\{P_{n}^{1}, P_{n}^{2}, P_{n}^{3}\right\}$ a family of supplementary projections sequences with the property

$$
P_{n+1}^{i} P_{n}^{i}=P_{n}^{i}, \quad \forall n \in \mathbb{N}, i=1,2,3 .
$$

Linear discrete-time system $(\mathscr{A})$ is given by

$$
A_{n}=\frac{u_{n}}{u_{n+1}} P_{n}^{1}+\frac{u_{n+1}}{u_{n}} P_{n}^{2}+\frac{v_{n}}{v_{n+1}} P_{n}^{3},
$$

where $u_{n}=e^{5 n /\left(2+w_{n}\right)}$ and $v_{n}=e^{4 n /\left(4+w_{n}\right)}$ and $w_{n}=\{6,7,8,6$, $7,8, \ldots\}$ is a periodic sequence. 
We have that $\mathscr{P}$ is invariant for $(\mathscr{A})$ and

$$
A_{m}^{n}=\frac{u_{n}}{u_{m}} P_{n}^{1}+\frac{u_{m}}{u_{n}} P_{n}^{2}+\frac{v_{n}}{v_{m}} P_{n}^{3},
$$

for all $(m, n) \in \Delta$.

A simple computation shows that for $h_{n}=e^{n \alpha}, k_{n}=e^{n \beta}$ with $\alpha \in(0,1 / 2), \beta \in(0,1 / 3)$, and $s_{n}=e^{n / 2}$ the pair $(\mathscr{A}, \mathscr{P})$ is $(h, k)$-trichotomic.

If we suppose that $(\mathscr{A}, \mathscr{P})$ is uniformly $(h, k)$-trichotomic, then, for $h_{n}=e^{n \alpha}, m=4 k+1, n=4 k, w_{n}=6$, and $w_{m}=8$, we obtain

$$
e^{\alpha} e^{k+1} \leq M
$$

which is a contradiction.

\section{4. $(h, k)$-Trichotomy of Datko Type}

Let $\left(h_{n}\right)$ be a growth rate which satisfies hypothesis $(\mathscr{H})$ and let $\left(f_{n}\right)$ be a growth rate given by Definition 2 .

We consider a pair $(\mathscr{A}, \mathscr{P})$, where $\mathscr{P}=\left\{P_{n}^{1}, P_{n}^{2}, P_{n}^{3}\right\}$ is a family of supplementary and invariant projections sequences for $(\mathscr{A})$.

The following result emphasizes a necessary condition for $(h, k)$-trichotomy.

Theorem 13. If $(\mathscr{A}, \mathscr{P})$ is $(h, k)$-trichotomic and $\left(h_{n}\right)$ satisfies hypothesis $(\mathscr{H})$ then there exist a growth rate $\left(g_{n}\right)$ and a nondecreasing sequence $\left(d_{n}\right), d_{n} \geq 1$, such that

$\left(f D_{1}\right)$

$$
\sum_{j=n+1}^{+\infty} f_{j}\left\|A_{j}^{n} P_{n}^{1} x\right\| \leq d_{n} f_{n}\left\|P_{n}^{1} x\right\|, \quad \forall(n, x) \in \mathbb{N} \times X ;
$$

$\left(f D_{2}\right)$

$$
\begin{aligned}
\sum_{j=n}^{m-1} \frac{1}{f_{j}}\left\|A_{j}^{n} P_{n}^{2} x\right\| \leq \frac{d_{m}}{f_{m}}\left\|A_{m}^{n} P_{n}^{2} x\right\|, & \\
& \forall(m, n, x) \in \Delta \times X, m>n ;
\end{aligned}
$$

$\left(g D_{3}\right)$

$$
\sum_{j=n+1}^{+\infty} \frac{1}{g_{j}}\left\|A_{j}^{n} P_{n}^{3} x\right\| \leq \frac{d_{n}}{g_{n}}\left\|P_{n}^{3} x\right\|, \quad \forall(n, x) \in \mathbb{N} \times X ;
$$

$\left(g D_{4}\right)$

$$
\begin{aligned}
\sum_{j=n}^{m-1} g_{j}\left\|A_{j}^{n} P_{n}^{3} x\right\| \leq g_{m} d_{m}\left\|A_{m}^{n} P_{n}^{3} x\right\|, & \\
& \forall(m, n, x) \in \Delta \times X, m>n .
\end{aligned}
$$

Proof. It is easy to see that, for

$$
\begin{gathered}
d_{n}=M \frac{h_{n} s_{n}}{f_{n}}, \\
g_{n}=\frac{h_{n} k_{n}}{f_{n}},
\end{gathered}
$$

where $M \in(1,+\infty),\left(f_{n}\right)$ are given by Definition 2 , and $\left(s_{n}\right)$, $\left(k_{n}\right)$ are given by Definition 9 , relations $\left(f D_{1}\right),\left(f D_{2}\right),\left(g D_{3}\right)$, and $\left(g D_{4}\right)$ are satisfied.

A necessary condition for polynomial trichotomy is represented by the following.

Corollary 14. If the pair $(\mathscr{A}, \mathscr{P})$ is polynomially trichotomic, then there are a nondecreasing sequence $\left(d_{n}\right), d_{n} \geq 1$, and two constants $a, b>0$ such that

$\left(p D_{1}\right)$

$$
\sum_{j=n+1}^{+\infty}(j+1)^{a}\left\|A_{j}^{n} P_{n}^{1} x\right\| \leq d_{n}(n+1)^{a}\left\|P_{n}^{1} x\right\|,
$$

$$
\forall(n, x) \in \mathbb{N} \times X
$$

$\left(p D_{2}\right)$

$$
\begin{aligned}
\sum_{j=n}^{m-1}(j+1)^{-a}\left\|A_{j}^{n} P_{n}^{2} x\right\| \leq & d_{m}(m+1)^{-a}\left\|A_{m}^{n} P_{n}^{2} x\right\|, \\
& \forall(m, n, x) \in \Delta \times X, m>n ;
\end{aligned}
$$

$\left(p D_{3}\right)$

$$
\begin{aligned}
& \sum_{j=n+1}^{+\infty}(j+1)^{-b}\left\|A_{j}^{n} P_{n}^{3} x\right\| \leq d_{n}(n+1)^{-b}\left\|P_{n}^{3} x\right\|, \\
& \forall(n, x) \in \mathbb{N} \times X ;
\end{aligned}
$$

$\left(p D_{4}\right)$

$$
\begin{aligned}
\sum_{j=n}^{m-1}(j+1)^{b}\left\|A_{j}^{n} P_{n}^{3} x\right\| \leq d_{m}(m+1)^{b}\left\|A_{m}^{n} P_{n}^{3} x\right\|, \\
\forall(m, n, x) \in \Delta \times X, m>n .
\end{aligned}
$$

Proof. It results from Theorem 13.

Definition 15 . One says that the pair $(\mathscr{A}, \mathscr{P})$ admits a $(h, k)$ trichotomy of Datko type if there exists a nondecreasing sequence $\left(d_{n}\right)$ with $d_{n} \geq 1$ such that

$\left(h D_{1}\right)$

$$
\sum_{j=n+1}^{+\infty} h_{j}\left\|A_{j}^{n} P_{n}^{1} x\right\| \leq d_{n} h_{n}\left\|P_{n}^{1} x\right\|, \quad \forall(n, x) \in \mathbb{N} \times X ;
$$

$\left(h D_{2}\right)$

$$
\sum_{j=n}^{m-1} \frac{1}{h_{j}}\left\|A_{j}^{n} P_{n}^{2} x\right\| \leq \frac{d_{m}}{h_{m}}\left\|A_{m}^{n} P_{n}^{2} x\right\|
$$

$\left(k D_{3}\right)$

$$
\forall(m, n, x) \in \Delta \times X, m>n ;
$$

$$
\sum_{j=n+1}^{+\infty} \frac{1}{k_{j}}\left\|A_{j}^{n} P_{n}^{3} x\right\| \leq \frac{d_{n}}{k_{n}}\left\|P_{n}^{3} x\right\|, \quad \forall(n, x) \in \mathbb{N} \times X ;
$$


$\left(k D_{4}\right)$

$$
\sum_{j=n}^{m-1} k_{j}\left\|A_{j}^{n} P_{n}^{3} x\right\| \leq k_{m} d_{m}\left\|A_{m}^{n} P_{n}^{3} x\right\|,
$$

$\forall(m, n, x) \in \Delta \times X, m>n$.

As particular cases, we mention the following:

(i) if $h_{n}=e^{n \alpha}$ with $\alpha>0$ and $k_{n}=e^{n \beta}$ with $\beta>0$ we obtain the notion of exponential trichotomy of Datko type;

(ii) if $h_{n}=(n+1)^{\alpha}$ with $\alpha>1$ and $k_{n}=(n+1)^{\beta}$ with $\beta>$ 1 we recover the concept of polynomial trichotomy of Datko type.

Remark 16. Theorem 13 emphasizes that if $\left(h_{n}\right),\left(k_{n}\right)$ are two growth rates, $\left(h_{n}\right)$ satisfies hypothesis $(\mathscr{H})$, and the pair $(\mathscr{A}, \mathscr{P})$ is $(h, k)$-trichotomic, then $(\mathscr{A}, \mathscr{P})$ admits a $(f, g)$ trichotomy of Datko type.

Theorem 17. If the pair $(\mathscr{A}, \mathscr{P})$ admits a $(h, k)$-trichotomy of Datko type, then $(\mathscr{A}, \mathscr{P})$ is $(h, k)$-trichotomic.

Proof. $\left(h t_{1}\right)$ Using condition $\left(h D_{1}\right)$ we obtain

$$
h_{m}\left\|A_{m}^{n} P_{n}^{1} x\right\| \leq h_{n} d_{n}\left\|P_{n}^{1} x\right\|, \quad \forall(m, n, x) \in \Delta \times X .
$$

$\left(h t_{2}\right)$ Similarly, by $\left(h D_{2}\right)$ for $j=n$ we have

$$
\begin{aligned}
& h_{m}\left\|P_{n}^{2} x\right\| \leq h_{n} d_{m}\left\|A_{m}^{n} P_{n}^{2} x\right\|, \\
& \forall(m, n, x) \in \Delta \times X, m>n .
\end{aligned}
$$

Obviously, the relation is valid for $m=n$.

$\left(k t_{3}\right)$ Inequality $\left(k D_{3}\right)$ implies that

$$
\begin{aligned}
& k_{n}\left\|A_{m}^{n} P_{n}^{3} x\right\| \leq d_{n} k_{m}\left\|P_{n}^{3} x\right\|, \quad \forall(m, n, x) \in \Delta \times X . \\
& \left(k t_{4}\right) \text { By }\left(k D_{4}\right)(\text { for } j=n) \text { we deduce } \\
& k_{n}\left\|P_{n}^{3} x\right\| \leq k_{m} d_{m}\left\|A_{m}^{n} P_{n}^{3} x\right\|, \\
& \forall(m, n, x) \in \Delta \times X, m>n,
\end{aligned}
$$

and the inequality is verified for $m=n$.

So, the pair $(\mathscr{A}, \mathscr{P})$ is $(h, k)$-trichotomic.

Corollary 18. If there exist two constants $a, b>1$ and $a$ nondecreasing sequence $\left(d_{n}\right), d_{n} \geq 1$, such that conditions $\left(p D_{1}\right)$ and $\left(p D_{3}\right)\left(\left(p D_{2}\right)\right.$ and $\left(p D_{4}\right)$, resp. $)$ from Corollary 14 are fulfilled for all $(n, x) \in \mathbb{N} \times X$ (for all $(m, n, x) \in \Delta \times X$, resp.) with $m>n$ then $(\mathscr{A}, \mathscr{P})$ is polynomially trichotomic.

Proof. It results from Theorem 17 for $h_{n}=(n+1)^{a}$ with $a>1$ and $k_{n}=(n+1)^{b}$, with $b>1$.

The following result represents a characterization for the exponential trichotomy.
Corollary 19. The pair $(\mathscr{A}, \mathscr{P})$ is exponentially trichotomic if and only if there exist the constants $a, b>0$ and $a$ nondecreasing sequence $\left(d_{n}\right), d_{n} \geq 1$, with the following properties:

$\left(e D_{1}\right)$

$$
\sum_{j=n+1}^{+\infty} e^{j a}\left\|A_{j}^{n} P_{n}^{1} x\right\| \leq d_{n} e^{n a}\left\|P_{n}^{1} x\right\|, \quad \forall(n, x) \in \mathbb{N} \times X ;
$$

$\left(e D_{2}\right)$

$$
\begin{aligned}
& \sum_{j=n}^{m-1} e^{-j a}\left\|A_{j}^{n} P_{n}^{2} x\right\| \leq d_{m} e^{-m a}\left\|A_{m}^{n} P_{n}^{2} x\right\|, \\
& \forall(m, n, x) \in \Delta \times X, m>n ;
\end{aligned}
$$

$\left(e D_{3}\right)$

$$
\sum_{j=n+1}^{+\infty} e^{-b j}\left\|A_{j}^{n} P_{n}^{3} x\right\| \leq d_{n} e^{-n b}\left\|P_{n}^{3} x\right\|,
$$

$$
\forall(n, x) \in \mathbb{N} \times X
$$

$\left(e D_{4}\right)$

$$
\begin{aligned}
\sum_{j=n}^{m-1} e^{j b}\left\|A_{j}^{n} P_{n}^{3} x\right\| \leq d_{m} e^{m b} & \left\|A_{m}^{n} P_{n}^{3} x\right\|, \\
& \forall(m, n, x) \in \Delta \times X, m>n .
\end{aligned}
$$

Proof.

Necessity. It is a particular case of Theorem 13 for

$$
\begin{aligned}
& h_{n}=e^{n \alpha}, \\
& f_{n}=e^{n a} \\
& \quad \text { with } a \in(0, \alpha), \\
& k_{n}=e^{n \beta} \quad \text { with } \beta>0, \\
& g_{n}=e^{n b}, \quad b=\beta+\alpha-a .
\end{aligned}
$$

Sufficiency. Using Theorem 17, for $h_{j}=e^{j a}$ and $k_{j}=e^{j b}$ we obtain that $(\mathscr{A}, \mathscr{P})$ is exponentially trichotomic.

Remark 20. The previous result shows that the exponential trichotomy and the exponential trichotomy of Datko type are equivalent.

\section{Lyapunov Functions for $(h, k)$-Trichotomy}

Throughout this section, $\left(h_{n}\right),\left(k_{n}\right)$ represent two growth rates, $(\mathscr{A})$ is a linear discrete-time system, and $\mathscr{P}=$ $\left\{P_{n}^{1}, P_{n}^{2}, P_{n}^{3}\right\}$ is a family of supplementary and invariant projections sequences for $(\mathscr{A})$. 
Definition 21. Two mappings $L_{1}, L_{2}: \Delta \times X \rightarrow \mathbb{R}_{+}$are called $(h, k)$-Lyapunov functions for the pair $(\mathscr{A}, \mathscr{P})$ if there exists a nondecreasing sequence $\left(t_{n}\right), t_{n} \geq 1$, such that

$$
\begin{aligned}
& \left(h L_{1}^{1}\right) \\
& L_{1}\left(m, p, P_{p}^{1} x\right)+\sum_{j=n+1}^{m} \frac{h_{j}}{h_{n}}\left\|A_{j}^{p} P_{p}^{1} x\right\| \leq L_{1}\left(n, p, P_{p}^{1} x\right) \\
& \quad \leq t_{p}\left\|P_{p}^{1} x\right\| ; \\
& \left(k L_{1}^{3}\right) \\
& \quad \sum_{j=n+1}^{m} \frac{k_{p}}{k_{j}}\left\|A_{j}^{p} P_{p}^{3} x\right\| \leq L_{1}\left(n, p, P_{p}^{3} x\right) \leq t_{p}\left\|P_{p}^{3} x\right\| ; \\
& \left(h L_{2}^{2}\right) \\
& L_{2}\left(n, p, P_{p}^{2} x\right)+\sum_{j=n}^{m-1} \frac{h_{m}}{h_{j}}\left\|A_{j}^{p} P_{p}^{2} x\right\| \leq L_{2}\left(m, p, P_{p}^{2} x\right) \\
& \quad \leq t_{m}\left\|A_{m}^{p} P_{p}^{2} x\right\| ; \\
& \left(k L_{2}^{3}\right) \\
& \sum_{j=n}^{m-1} \frac{k_{j}}{k_{m}}\left\|A_{j}^{p} P_{p}^{3} x\right\| \leq L_{2}\left(m, p, P_{p}^{3} x\right) \leq t_{m}\left\|A_{m}^{p} P_{p}^{3} x\right\|,
\end{aligned}
$$

for all $(m, n, p) \in \mathbb{N}^{3}$ with $m>n \geq p$ and for all $x \in X$.

In particular, if

(i) $h_{n}=e^{n \alpha}$, with $\alpha>0, k_{n}=e^{n \beta}$, with $\beta>0$ then the $(h, k)$-Lyapunov functions are called exponential Lyapunov functions;

(ii) $h_{n}=(n+1)^{\alpha}$, with $\alpha>0, k_{n}=(n+1)^{\beta}$, with $\beta>0$ then the $(h, k)$-Lyapunov functions are called polynomial Lyapunov functions.

Example 22. On $X=l^{\infty}(\mathbb{N}, \mathbb{R})$, the Banach space of bounded real-valued sequences, endowed with the norm

$$
\|x\|_{\infty}=\sup _{n \in \mathbb{N}}\left|x_{n}\right|, \quad x=\left(x_{n}\right)_{n \in \mathbb{N}} \in X,
$$

we consider $\mathscr{P}=\left\{P_{n}^{1}, P_{n}^{2}, P_{n}^{3}\right\}$, with

$$
\begin{aligned}
& P_{n}^{1} x=\chi_{3 \mathbb{N}} x, \\
& P_{n}^{2} x=\chi_{3 \mathbb{N}+1} x, \\
& P_{n}^{3} x=\chi_{3 \mathbb{N}+2} x,
\end{aligned}
$$

where $\chi_{A}$ represents the characteristic function of set $A$.

Also, linear discrete-time system $(\mathscr{A})$ is defined by

$$
A_{n}=e^{3 n-3(n+1)} P_{n}^{1}+e^{3(n+1)-3 n} P_{n}^{2}+P_{n}^{3}
$$

and we have that

$$
A_{m}^{n}=e^{3 n-3 m} P_{n}^{1}+e^{3 m-3 n} P_{n}^{2}+P_{n}^{3},
$$

for all $(m, n) \in \Delta$.
For the growth rates $h_{n}=e^{2 n}$ and $k_{n}=e^{4 n}$ we define the exponential Lyapunov functions:

$$
\begin{aligned}
& L_{1}(m, n, x)=\sum_{j=m+1}^{+\infty} e^{2 j-2 n}\left\|A_{j}^{n} P_{n}^{1} x\right\|+\sum_{j=m+1}^{+\infty} e^{4 n-4 j}\left\|A_{j}^{n} P_{n}^{3} x\right\|, \\
& L_{2}(m, n, x) \\
& = \begin{cases}\sum_{j=n}^{m-1} e^{2 m-2 j}\left\|A_{j}^{n} P_{n}^{2} x\right\|+\sum_{j=n}^{m-1} e^{4 j-4 m}\left\|A_{j}^{n} P_{n}^{3} x\right\|, & \text { if } m>n \\
0, & \text { if } m=n .\end{cases}
\end{aligned}
$$

After some computations, we obtain that for $t_{n}=e^{4(n+1)} /\left(e^{4}-\right.$ $1)$ the mappings $L_{1}$ and $L_{2}$ are exponential Lyapunov functions for the pair $(\mathscr{A}, \mathscr{P})$.

In the following, we give a characterization for the $(h, k)$-trichotomy of Datko type in terms of $(h, k)$-Lyapunov functions.

Theorem 23. The pair $(\mathscr{A}, \mathscr{P})$ admits a $(h, k)$-trichotomy of Datko type if and only if there exist two $(h, k)$-Lyapunov functions for $(\mathscr{A}, \mathscr{P})$.

Proof.

Necessity. Let $L_{1}, L_{2}: \Delta \times X \rightarrow \mathbb{R}_{+}$, defined by

$$
\begin{aligned}
& L_{1}(m, n, x)=\sum_{j=m+1}^{+\infty} \frac{h_{j}}{h_{n}}\left\|A_{j}^{n} P_{n}^{1} x\right\|+\sum_{j=m+1}^{+\infty} \frac{k_{n}}{k_{j}}\left\|A_{j}^{n} P_{n}^{3} x\right\|, \\
& L_{2}(m, n, x) \\
& =\left\{\begin{array}{lr}
\sum_{j=n}^{m-1} \frac{h_{m}}{h_{j}}\left\|A_{j}^{n} P_{n}^{2} x\right\|+\sum_{j=n}^{m-1} \frac{k_{j}}{k_{m}}\left\|A_{j}^{n} P_{n}^{3} x\right\|, & \text { if } m>n \\
0, & \text { if } m=n,
\end{array}\right.
\end{aligned}
$$

respectively. Then

$$
\begin{aligned}
& \left(h L_{1}^{1}\right) \\
& L_{1}\left(m, p, P_{p}^{1} x\right)+\sum_{j=n+1}^{m} \frac{h_{j}}{h_{n}}\left\|A_{j}^{p} P_{p}^{1} x\right\| \\
& \quad=\sum_{j=m+1}^{+\infty} \frac{h_{j}}{h_{p}}\left\|A_{j}^{p^{p}} P_{p}^{1} x\right\|+\sum_{j=n+1}^{m} \frac{h_{j}}{h_{n}}\left\|A_{j}^{p_{j}} P_{p}^{1} x\right\| \\
& \quad \leq \sum_{j=n+1}^{+\infty} \frac{h_{j}}{h_{p}}\left\|A_{j}^{p} P_{p}^{1} x\right\|=L_{1}\left(n, p, P_{p}^{1} x\right) \leq d_{p}\left\|P_{p}^{1} x\right\| ; \\
& \left(k L_{1}^{3}\right) \sum_{j=n+1}^{m} \frac{k_{p}}{k_{j}}\left\|A_{j}^{p} P_{p}^{3} x\right\| \leq \sum_{j=n+1}^{+\infty} \frac{k_{p}}{k_{j}}\left\|A_{j}^{p} P_{p}^{3} x\right\| \\
& =L_{1}\left(n, p, P_{p}^{3} x\right) \leq d_{p}\left\|P_{p}^{3} x\right\| ;
\end{aligned}
$$


$\left(h L_{2}^{2}\right)$

$$
\begin{aligned}
& L_{2}\left(n, p, P_{p}^{2} x\right)+\sum_{j=n}^{m-1} \frac{h_{m}}{h_{j}}\left\|A_{j}^{p} P_{p}^{2} x\right\| \\
& \quad=\sum_{j=p}^{n-1} \frac{h_{n}}{h_{j}}\left\|A_{j}^{p} P_{p}^{2} x\right\|+\sum_{j=n}^{m-1} \frac{h_{m}}{h_{j}}\left\|A_{j}^{p} P_{p}^{2} x\right\| \\
& \leq \sum_{j=p}^{m-1} \frac{h_{m}}{h_{j}}\left\|A_{j}^{p} P_{p}^{2} x\right\|=L_{2}\left(m, p, P_{p}^{2} x\right) \\
& \leq d_{m}\left\|A_{m}^{p} P_{p}^{2} x\right\| ;
\end{aligned}
$$

$\left(k L_{2}^{3}\right)$

$$
\begin{aligned}
\sum_{j=n}^{m-1} \frac{k_{j}}{k_{m}}\left\|A_{j}^{p} P_{p}^{3} x\right\| & \leq \sum_{j=p}^{m-1} \frac{k_{j}}{k_{m}}\left\|A_{j}^{p} P_{p}^{3} x\right\|=L_{2}\left(m, p, P_{p}^{3} x\right) \\
& \leq d_{m}\left\|A_{m}^{p} P_{p}^{3} x\right\|,
\end{aligned}
$$

for all $(m, n, p) \in \mathbb{N}^{3}$ with $m>n \geq p$ and for all $x \in X$.

Sufficiency. $\left(h D_{1}\right)$ From $\left(h L_{1}^{1}\right)$, for $p=n$ we obtain

$$
\sum_{j=n+1}^{m} \frac{h_{j}}{h_{n}}\left\|A_{j}^{n} P_{n}^{1} x\right\| \leq t_{n}\left\|P_{n}^{1} x\right\|,
$$

and for $m \rightarrow+\infty$ it results in condition $\left(h D_{1}\right)$.

$\left(h D_{2}\right)$ For $p=n$ in $\left(h L_{2}^{2}\right)$ we deduce

$$
\sum_{j=n}^{m-1} \frac{h_{m}}{h_{j}}\left\|A_{j}^{n} P_{n}^{2} x\right\| \leq t_{m}\left\|A_{m}^{n} P_{n}^{2} x\right\|,
$$

for all $(m, n) \in \Delta, m>n, x \in X$.

$\left(k D_{3}\right)$ Putting $p=n$ in $\left(k L_{1}^{3}\right)$ we obtain

$$
\sum_{j=n+1}^{m} \frac{k_{n}}{k_{j}}\left\|A_{j}^{n} P_{n}^{3} x\right\| \leq t_{n}\left\|P_{n}^{3} x\right\|,
$$

and for $m \rightarrow+\infty$ it results in $\left(k D_{3}\right)$.

$\left(k D_{4}\right)$ By condition $\left(k L_{2}^{3}\right)$ it follows

$$
\sum_{j=n}^{m-1} \frac{k_{j}}{k_{m}}\left\|A_{j}^{n} P_{n}^{3} x\right\| \leq t_{m}\left\|A_{m}^{n} P_{n}^{3} x\right\|,
$$

for all $(m, n) \in \Delta, m>n, x \in X$.

Thus, it results in that $(\mathscr{A}, \mathscr{P})$ admits a $(h, k)$-trichotomy of Datko type.

A sufficient condition for $(h, k)$-trichotomy given through the Lyapunov functions is as follows.

Corollary 24. If there exist two $(h, k)$-Lyapunov functions $L_{1}, L_{2}$ for the pair $(\mathscr{A}, \mathscr{P})$, then $(\mathscr{A}, \mathscr{P})$ is $(h, k)$-trichotomic.

Proof. It is obtained from Theorems 17 and 23.
Corollary 25. Let $\left(h_{n}\right),\left(k_{n}\right)$ be two growth rates such that $\left(h_{n}\right)$ satisfies hypothesis $(\mathscr{H})$. If $(\mathscr{A}, \mathscr{P})$ is $(h, k)$-trichotomic then there exist two $(f, g)$-Lyapunov functions for $(\mathscr{A}, \mathscr{P})$, where $\left(f_{n}\right),\left(g_{n}\right)$ are the growth rates given by Definition 2 (Theorem 13, resp.).

Proof. It is immediate by Remark 16 and Theorem 23.

An important characterization for the exponential trichotomy in terms of Lyapunov functions is represented by the following.

Corollary 26. The pair $(\mathscr{A}, \mathscr{P})$ is exponentially trichotomic if and only if there exist $L_{1}, L_{2}$ two exponential Lyapunov functions for $(\mathscr{A}, \mathscr{P})$.

Proof. It results by Remark 20 and Theorem 23.

Corollary 27. If $(\mathscr{A}, \mathscr{P})$ is polynomially trichotomic, then there exist $L_{1}, L_{2}$ polynomial Lyapunov functions for $(\mathscr{A}, \mathscr{P})$.

Proof. It is a consequence of Corollary 25.

Corollary 28. If there exist two $(h, k)$-Lyapunov functions for the pair $(\mathscr{A}, \mathscr{P})$, where

$$
\begin{aligned}
& h_{n}=(n+1)^{\alpha}, \text { with } \alpha>1, \\
& k_{n}=(n+1)^{\beta}, \text { with } \beta>1,
\end{aligned}
$$

then $(\mathscr{A}, \mathscr{P})$ is polynomially trichotomic.

Proof. It results from Theorems 17 and 23 for

$$
\begin{aligned}
& h_{n}=(n+1)^{\alpha}, \quad \text { with } \alpha>1, \\
& k_{n}=(n+1)^{\beta}, \quad \text { with } \beta>1 .
\end{aligned}
$$

\section{Conflict of Interests}

The authors declare that there is no conflict of interests regarding the publication of this paper.

\section{References}

[1] A. I. Alonso, J. Hong, and R. Obaya, "Exponential dichotomy and trichotomy for difference equations," Computers \& Mathematics with Applications, vol. 38, no. 1, pp. 41-49, 1999.

[2] M.-G. Babuţia, M. I. Kovács, M. Lăpădat, and M. Megan, "Discrete $(h, k)$-dichotomy and remarks on the boundedness of the projections," Journal of Operators, vol. 2014, Article ID 196345, 6 pages, 2014.

[3] M. G. Babuţia, M. Megan, and I. L. Popa, "On ( $h, k)$-dichotomies for nonautonomous linear difference equations in Banach spaces," International Journal of Differential Equations, vol. 2013, Article ID 761680, 7 pages, 2013.

[4] L. Barreira and C. Valls, "Lyapunov functions for trichotomies with growth rates," Journal of Differential Equations, vol. 248, no. 1, pp. 151-183, 2010. 
[5] L. Barreira and C. Valls, "Lyapunov sequences for exponential trichotomies," Nonlinear Analysis: Theory, Methods \& Applications, vol. 72, no. 1, pp. 192-203, 2010.

[6] A. J. G. Bento and C. M. Silva, "Nonuniform $(\mu, \nu)$-dichotomies and local dynamics of difference equations," Nonlinear Analysis: Theory, Methods \& Applications, vol. 75, no. 1, pp. 78-90, 2012.

[7] C. Cuevas and C. Vidal, "Weighted exponential trichotomy of linear difference equations," Dynamics of Continuous, Discrete \& Impulsive Systems. Series A. Mathematical Analysis, vol. 15, no. 3, pp. 353-379, 2008.

[8] R. Datko, "Uniform asymptotic stability of evolutionary processes in a Banach space," SIAM Journal on Mathematical Analysis, vol. 3, no. 3, pp. 428-445, 1972.

[9] S. Elaydi and O. Hajek, "Exponential trichotomy of differential systems," Journal of Mathematical Analysis and Applications, vol. 129, no. 2, pp. 362-374, 1988.

[10] S. Elaydi and O. Hajek, "Exponential dichotomy and trichotomy of nonlinear differential equations," Differential Integral Equations, vol. 3, no. 6, pp. 1201-1224, 1990.

[11] S. Elaydi and K. Janglajew, "Dichotomy and trichotomy of difference equations," Journal of Difference Equations and Applications, vol. 3, no. 5-6, pp. 417-448, 1998.

[12] Z.-G. Li, X.-Q. Song, and X.-L. Yang, "On nonuniform polynomial trichotomy of linear discrete-time systems in Banach spaces," Journal of Applied Mathematics, vol. 2014, Article ID 807265, 6 pages, 2014.

[13] J. López-Fenner and M. Pinto, " $h, k)$-Trichotomies and asymptotics of nonautonomous difference systems," Computers \& Mathematics with Applications, vol. 33, no. 10, pp. 105-124, 1997.

[14] M. Megan and L. Buliga, "Nonuniform exponential trichotomy for difference equations in Banach spaces," Hot Topics in Operator Theory, Theta Series in Advanced Mathematics, vol. 9, pp. 151-158, 2008.

[15] K. M. Przyłuski and S. Rolewicz, "On stability of linear timevarying infinite-dimensional discrete-time systems," Systems \& Control Letters, vol. 4, no. 5, pp. 307-315, 1984.

[16] I.-L. Popa, M. Megan, and T. Ceauşu, "Exponential dichotomies for linear discrete-time systems in Banach spaces," Applicable Analysis and Discrete Mathematics, vol. 6, no. 1, pp. 140-155, 2012.

[17] P. Preda and M. Megan, "Exponential dichotomy of evolutionary processes in Banach spaces," Czechoslovak Mathematical Journal, vol. 35, no. 110, pp. 312-323, 1985.

[18] B. Sasu, "On exponential dichotomy of variational difference equations," Discrete Dynamics in Nature and Society, vol. 2009, Article ID 324273, 18 pages, 2009.

[19] M. Megan and C. Stoica, "On uniform exponential trichotomy of evolution operators in Banach spaces," Integral Equations and Operator Theory, vol. 60, no. 4, pp. 499-506, 2008.

[20] M. Megan and C. Stoica, "Trichotomy for discrete skewevolution semiflows in Banach spaces," Annals of the Tiberiu Popoviciu Seminar of Functional Equations, Approximation and Convexity, vol. 5, pp. 79-85, 2007.

[21] G. Papaschinopoulos, "On exponential trichotomy of linear difference equations," Applicable Analysis, vol. 40, no. 2-3, pp. 89-109, 1991.

[22] I.-L. Popa, M. Megan, and T. Ceauşu, "On h-trichotomy of linear discrete-time systems in Banach spaces," Acta Universitatis Apulensis, vol. 39, pp. 329-339, 2014.

[23] A. L. Sasu and B. Sasu, "Input-output admissibility and exponential trichotomy of difference equations," Journal of Mathematical Analysis and Applications, vol. 380, no. 1, pp. 17-32, 2011.
[24] B. Sasu and A. L. Sasu, "Nonlinear criteria for the existence of the exponential trichotomy in infinite dimensional spaces," Nonlinear Analysis: Theory, Methods \& Applications, vol. 74, no. 15, pp. 5097-5110, 2011.

[25] G. Papaschinopoulos, "A characterization of exponential trichotomy via Lyapunov functions for difference equations," Mathematica Japonica, vol. 37, no. 3, pp. 555-562, 1992.

[26] I.-L. Popa, T. Ceauşu, and M. Megan, "Nonuniform power instability and Lyapunov sequences," Applied Mathematics and Computation, vol. 247, pp. 969-975, 2014. 


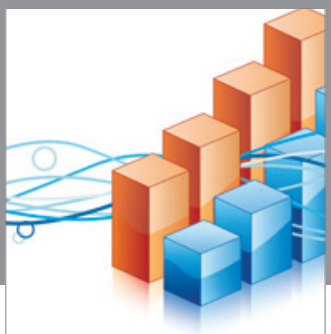

Advances in

Operations Research

vatem alat4

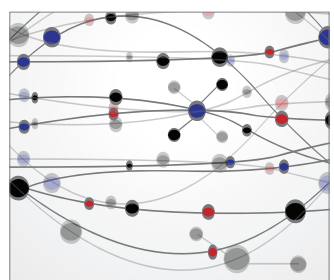

\section{The Scientific} World Journal
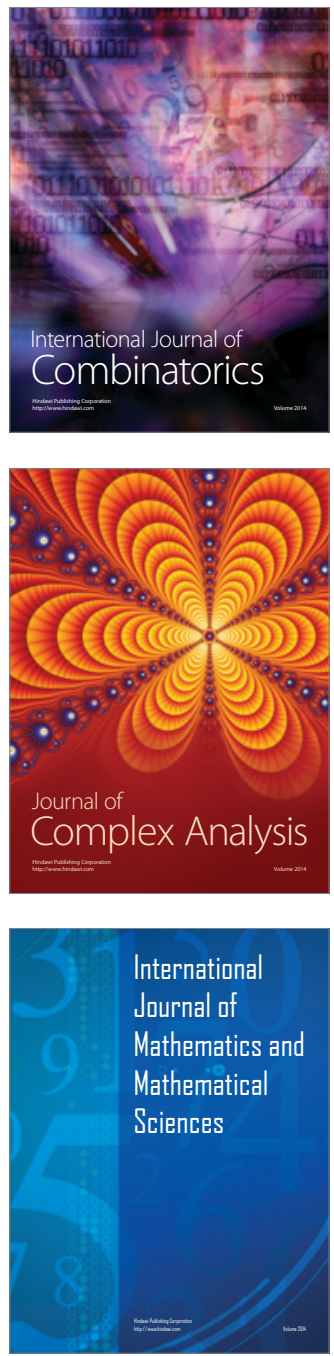
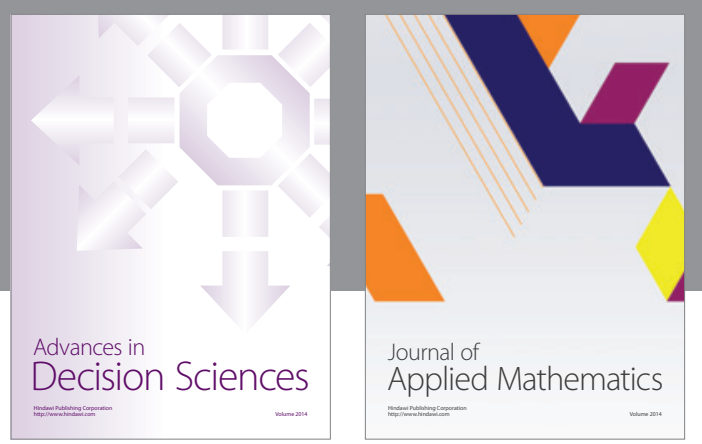

Algebra

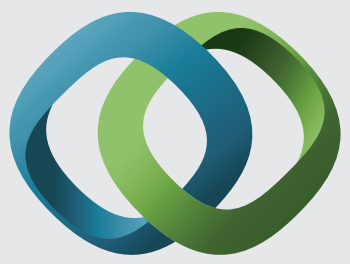

\section{Hindawi}

Submit your manuscripts at

http://www.hindawi.com
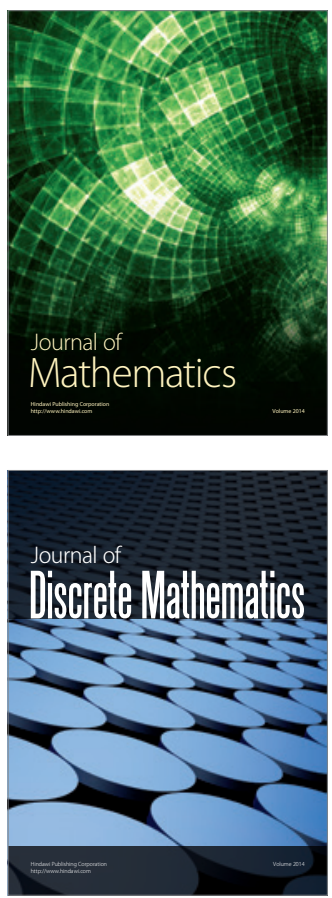

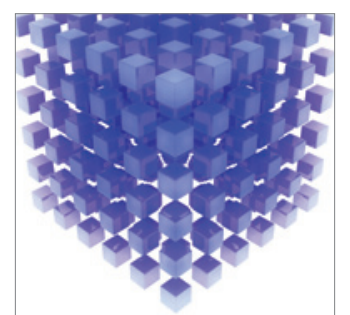

Mathematical Problems in Engineering
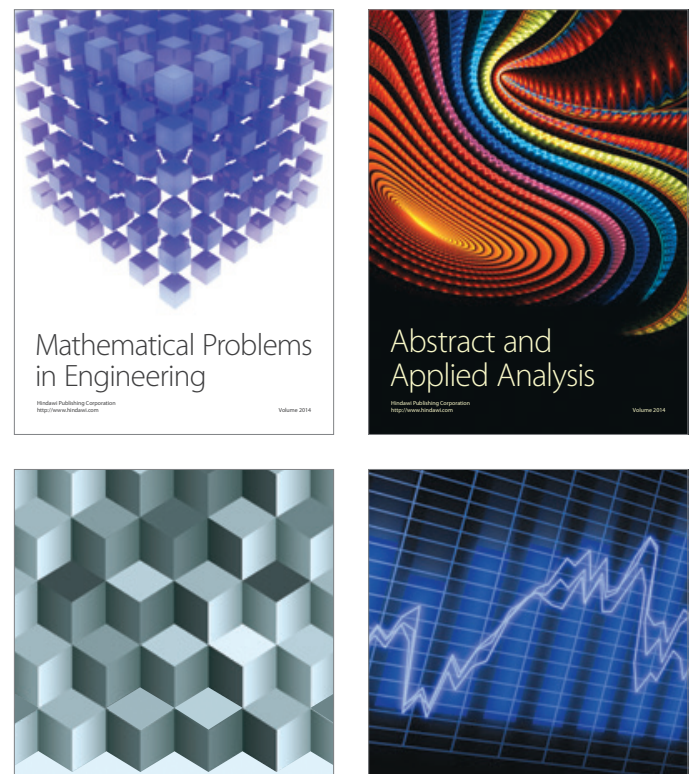

Journal of

Function Spaces

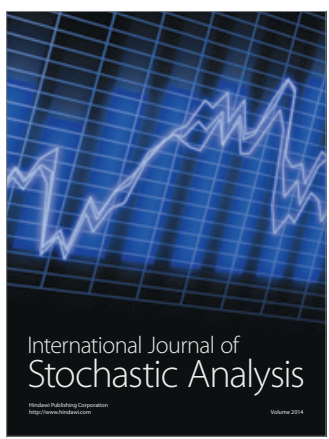

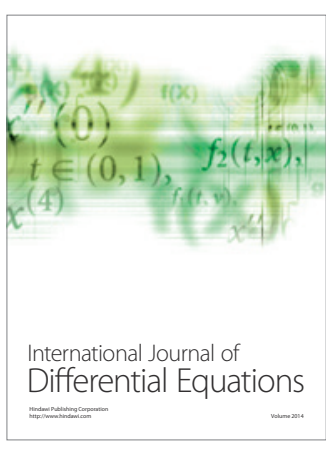
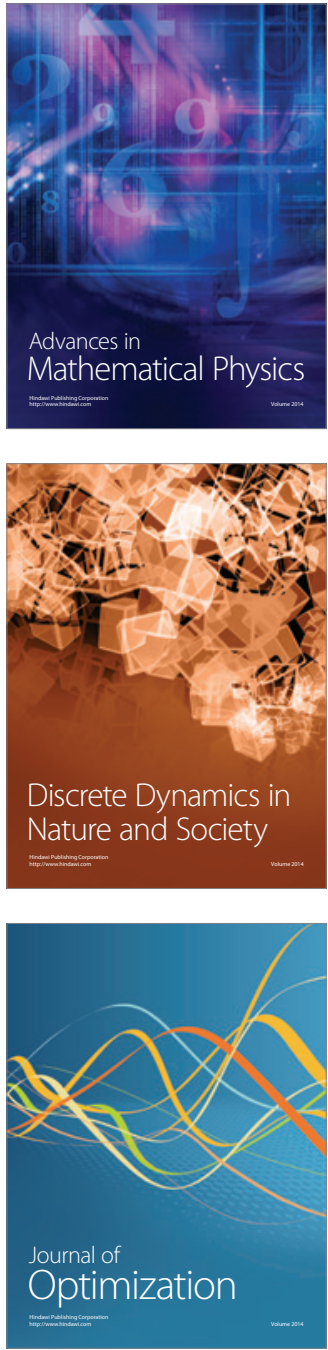